\title{
Effects of tensor correlations on low-lying collective states in finite nuclei
}

\author{
Li-Gang Cao (曹李刚), ${ }^{1,2,3}$ H. Sagawa, ${ }^{2}$ and G. Colò ${ }^{4,5}$ \\ ${ }^{1}$ Institute of Modern Physics, Chinese Academy of Science, Lanzhou 730000, People's Republic of China \\ ${ }^{2}$ Center for Mathematics and Physics, University of Aizu, Aizu-Wakamatsu, Fukushima 965-8560, Japan \\ ${ }^{3}$ Center of Theoretical Nuclear Physics, National Laboratory of Heavy Ion Accelerator of Lanzhou, Lanzhou 730000, \\ People's Republic of China \\ ${ }^{4}$ Dipartimento di Fisica, Università degli Studi di Milano, via Celoria 16, I-20133 Milano, Italy \\ ${ }^{5}$ Istituto Nazionale di Fisica Nucleare (INFN), Sez. di Milano, via Celoria 16, I-20133 Milano, Italy \\ (Received 4 January 2011; revised manuscript received 17 February 2011; published 29 March 2011)
}

\begin{abstract}
We present a systematic analysis of the effects induced by tensor correlations on low-lying collective states of magic nuclei, by using the fully self-consistent random phase approximation (RPA) model with Skyrme interactions. The role of the tensor correlations is analyzed in detail in the case of quadrupole $\left(2^{+}\right)$and octupole $\left(3^{-}\right)$low-lying collective states in ${ }^{208} \mathrm{~Pb}$. The example of ${ }^{40} \mathrm{Ca}$ is also discussed, as well as the case of magnetic dipole states $\left(1^{+}\right)$.
\end{abstract}

DOI: 10.1103/PhysRevC.83.034324

PACS number(s): 21.60.Jz, 21.65.Ef, 24.30.Cz, 24.30.Gd

\section{INTRODUCTION}

The nuclear effective interactions such as the zero-range Skyrme forces have been quite successful in describing different nuclear phenomena, and their performance is still improving due to changes in their form and their parameters. The Skyrme forces contain typically 10 free parameters that are fitted to reproduce empirical bulk properties of uniform nuclear matter and masses and radii of a few magic nuclei. These forces describe in a reasonable way the global features of the groundstate properties in a wide range of the nuclear chart (i.e., binding energies, radii, deformations). Properties of excited states (vibrations, rotations) have also been studied using the Skyrme interactions, allowing much physical insight [1].

Most of the Skyrme sets that have been widely used are purely central. Many groups have recently devoted much attention to the role played by the zero-range tensor terms that can be added to the standard Skyrme terms (see Refs. [2-9]). Many of these theoretical studies have followed the claim that the tensor force is crucial for the understanding of the evolution of the single-particle energies in exotic nuclei $[4,10]$. While the mentioned studies have elucidated that the tensor force does play a role on single-particle states, these are probably not the right observables on which to constrain an effective force or an effective functional, since other correlations beyond mean field are expected to manifest themselves.

The study of nuclear collective vibrations, within selfconsistent random phase approximation (RPA) on top of Hartree-Fock (HF), is a well-defined framework that has allowed testing the effective Skyrme sets for many years. Recently, fully self-consistent RPA as well as quasiparticle RPA [11] schemes have been developed including the twobody spin-orbit and Coulomb interactions (whose contribution to the residual interaction had been previously been dropped). In Ref. [12] we extended the fully self-consistent RPA by also including tensor terms both in HF and in RPA, and we analyzed the results in the case of the multipole response of magic nuclei.
In this paper we study systematically the effect of the tensor interactions on the low-lying collective states in ${ }^{208} \mathrm{~Pb}$ by employing a very wide range of Skyrme interactions with tensor terms. We carefully check the interaction dependence of the excitation energies and the transition strengths. Our goal is to elucidate how realistic is the description of these important nuclear properties, and if we can get some kind of improvement by adding tensor terms on top of the standard central Skyrme interactions. Proper descriptions of collective excitations are quite important for further applications of the microscopic particle-vibration coupling models [13].

The outline of the present paper is the following. We will survey very briefly our model in Sec. II. The discussion of the results for quadrupole, octupole, and magnetic dipole states is given in Sec. III. A summary, together with some perspectives for future work on the tensor force and on possible constraints on its parameters, is given in Sec. IV.

\section{FORMALISM}

We employ the triplet-even and triplet-odd zero-range tensor force, which originated from the form given by T. H. R. Skyrme [14] and read

$$
\begin{aligned}
V^{T}= & \frac{T}{2}\left\{\left[\left(\sigma_{1} \cdot \mathbf{k}^{\prime}\right)\left(\boldsymbol{\sigma}_{2} \cdot \mathbf{k}^{\prime}\right)-\frac{1}{3}\left(\sigma_{1} \cdot \sigma_{2}\right) \mathbf{k}^{\prime 2}\right] \delta\left(\mathbf{r}_{1}-\mathbf{r}_{2}\right)\right. \\
& \left.+\delta\left(\mathbf{r}_{1}-\mathbf{r}_{2}\right)\left[\left(\sigma_{1} \cdot \mathbf{k}\right)\left(\sigma_{2} \cdot \mathbf{k}\right)-\frac{1}{3}\left(\sigma_{1} \cdot \sigma_{2}\right) \mathbf{k}^{2}\right]\right\} \\
& +\frac{U}{2}\left\{\left(\sigma_{1} \cdot \mathbf{k}^{\prime}\right) \delta\left(\mathbf{r}_{1}-\mathbf{r}_{2}\right)\left(\sigma_{2} \cdot \mathbf{k}\right)+\left(\sigma_{2} \cdot \mathbf{k}^{\prime}\right) \delta\left(\mathbf{r}_{1}-\mathbf{r}_{2}\right)\right. \\
& \left.\times\left(\sigma_{1} \cdot \mathbf{k}\right)-\frac{2}{3}\left[\left(\sigma_{1} \cdot \boldsymbol{\sigma}_{2}\right) \mathbf{k}^{\prime} \cdot \delta\left(\mathbf{r}_{1}-\mathbf{r}_{2}\right) \mathbf{k}\right]\right\}
\end{aligned}
$$

In the above expression, the operator $\mathbf{k}=\left(\nabla_{1}-\nabla_{2}\right) / 2 i$ acts on the right, and $\mathbf{k}^{\prime}=-\left(\nabla_{1}^{\prime}-\nabla_{2}^{\prime}\right) / 2 i$ acts on the left. The coupling constants $T$ and $U$ denote the strengths of the triplet-even and triplet-odd tensor interactions, respectively. 
The central exchange and tensor contributions to the energy density $H(r)$ are

$$
\Delta H=\frac{1}{2} \alpha\left(J_{n}^{2}+J_{p}^{2}\right)+\beta J_{n} J_{p},
$$

where $J_{n}$ and $J_{p}$ are spin-orbit densities for neutrons and protons, respectively, defined by

$$
J_{q}(r)=\frac{1}{4 \pi r^{3}} \sum_{i}\left(2 j_{i}+1\right)\left[j_{i}\left(j_{i}+1\right)-l_{i}\left(l_{i}+1\right)-\frac{3}{4}\right] R_{i}^{2}(r) .
$$

In Eq. (3), the isospin quantum number $q=n(p)$ labels neutrons (protons), whereas the index $i=n, l, j$ runs over all occupied states having the given $q$, and $R_{i}(r) \equiv u_{i}(r) / r$ is the radial part of the wavefunction. The spin-orbit potential is given by

$$
U_{\mathrm{so}}^{(q)}=\frac{W_{0}}{2 r}\left(2 \frac{d \rho_{q}}{d r}+\frac{d \rho_{q^{\prime}}}{d r}\right)+\left(\alpha \frac{J_{q}}{r}+\beta \frac{J_{q^{\prime}}}{r}\right),
$$

where the first term comes from the Skyrme two-body spinorbit interaction, whereas the second term includes both the central exchange and the tensor contributions, that is,

$$
\alpha=\alpha_{C}+\alpha_{T}, \quad \beta=\beta_{C}+\beta_{T}
$$

with

$$
\begin{gathered}
\alpha_{C}=\frac{1}{8}\left(t_{1}-t_{2}\right)-\frac{1}{8}\left(t_{1} x_{1}-t_{2} x_{2}\right), \quad \beta_{C}=-\frac{1}{8}\left(t_{1} x_{1}+t_{2} x_{2}\right), \\
\alpha_{T}=\frac{5}{12} U, \quad \beta_{T}=\frac{5}{24}(T+U) .
\end{gathered}
$$

It should be noted that $J_{q}$ gives essentially no contribution in the spin-orbit saturated $(l \cdot s$ closed) nuclei in which the two spin-orbit partners are both occupied or unoccupied. Therefore, the tensor force gives a negligible contribution to the energy density in nuclei that are spin-orbit saturated for both neutrons and protons. We choose ${ }^{40} \mathrm{Ca}$ as an example of such nuclei. ${ }^{208} \mathrm{~Pb}$ is chosen as a complementary example since both protons and neutrons are spin-orbit unsaturated.

We adopt two different kinds of available parameter sets among the existing Skyrme interactions with tensor terms for the calculations presented below. On the one hand, we have chosen the sets SGII, SIII, and SLy5 and supplemented them with tensor terms characterized by the parameter values given in Refs. [4,5]. The second kind of choice is associated with the forces having all parameters (those characterizing the central terms as well as those associated with the tensor terms) fitted on an equal footing: In particular, we have performed calculations with all the sets of the TI $J$ families introduced in Ref. [6] and the parameter sets SKXTA and SKXTB in Ref. [2]. These TI $J$ interactions are defined by the fact that the parameters shown in Eq. (5) are fixed to be $\alpha=60(\mathrm{~J}-2) \mathrm{MeV} \cdot \mathrm{fm}^{5}$ and $\beta=60$ (I-2) $\mathrm{MeV} \cdot \mathrm{fm}^{5}$, respectively. The values of the parameters defining the tensor terms in all sets we have employed are given in Table I in order to make our later discussion easier.

As mentioned in Sec. I, Skyrme-RPA theory has been well known for many years, especially in its matrix formulation.

\begin{tabular}{|c|c|c|c|c|}
\hline & $T$ & $U$ & $\alpha$ & $\beta$ \\
\hline SLy5 & 888.0 & -408.0 & -89.8 & 51.1 \\
\hline SGII & 1008.0 & -432.0 & -122.3 & 130.0 \\
\hline SIII & 1008.0 & -432.0 & -118.7 & 120.0 \\
\hline SKXTA & 384.0 & 144.0 & 93.6 & 94.2 \\
\hline SKXTB & 811.2 & -283.2 & -83.9 & 96.1 \\
\hline $\mathrm{T} 11$ & 258.9 & -342.8 & -60.0 & -60.0 \\
\hline T12 & 116.4 & -198.2 & 0.0 & -60.0 \\
\hline T13 & -20.8 & -51.7 & 60.0 & -60.0 \\
\hline T14 & -165.4 & 92.5 & 120.0 & -60.0 \\
\hline $\mathrm{T} 15$ & -500.9 & 173.3 & 180.0 & -60.0 \\
\hline T16 & -646.2 & 314.7 & 240.0 & -60.0 \\
\hline $\mathrm{T} 21$ & 476.9 & -369.4 & -60.0 & 0.0 \\
\hline $\mathrm{T} 22$ & 356.1 & -217.5 & 0.0 & 0.0 \\
\hline $\mathrm{T} 23$ & 183.9 & -82.7 & 60.0 & 0.0 \\
\hline $\mathrm{T} 24$ & 33.7 & 59.2 & 120.0 & 0.0 \\
\hline $\mathrm{T} 25$ & -69.4 & 216.0 & 180.0 & 0.0 \\
\hline T26 & -209.7 & 362.1 & 240.0 & 0.0 \\
\hline T31 & 738.6 & -382.5 & -60.0 & 60.0 \\
\hline T32 & 613.1 & -231.5 & 0.0 & 60.0 \\
\hline T33 & 439.3 & -97.9 & 60.0 & 60.0 \\
\hline T34 & 246.6 & 30.8 & 120.0 & 60.0 \\
\hline T35 & 125.5 & 180.9 & 180.0 & 60.0 \\
\hline T36 & 27.2 & 341.8 & 240.0 & 60.0 \\
\hline T41 & 884.9 & -433.6 & -60.0 & 120.0 \\
\hline $\mathrm{T} 42$ & 730.7 & -292.9 & 0.0 & 120.0 \\
\hline $\mathrm{T} 43$ & 590.6 & -147.5 & 60.0 & 120.0 \\
\hline $\mathrm{T} 44$ & 520.9 & 21.5 & 120.0 & 120.0 \\
\hline $\mathrm{T} 45$ & 346.9 & 156.9 & 180.0 & 120.0 \\
\hline $\mathrm{T} 46$ & 249.6 & 314.6 & 240.0 & 120.0 \\
\hline T51 & 1179.9 & -435.7 & -60.0 & 180.0 \\
\hline T52 & 918.2 & -329.9 & 0.0 & 180.0 \\
\hline T53 & 974.9 & -119.1 & 60.0 & 180.0 \\
\hline T54 & 727.3 & -8.4 & 120.0 & 180.0 \\
\hline T55 & 564.6 & 129.3 & 180.0 & 180.0 \\
\hline T56 & 448.3 & 282.9 & 240.0 & 180.0 \\
\hline T61 & 1335.5 & -480.4 & -60.0 & 240.0 \\
\hline T62 & 1256.5 & -313.9 & 0.0 & 240.0 \\
\hline T63 & 1043.8 & -193.3 & 60.0 & 240.0 \\
\hline T64 & 1046.8 & -0.6 & 120.0 & 240.0 \\
\hline T65 & 823.2 & 119.7 & 180.0 & 240.0 \\
\hline T66 & 708.5 & 270.9 & 240.0 & 240.0 \\
\hline
\end{tabular}
We have been using for several applications a scheme that is fully self-consistent (see, e.g., Refs. $[15,16]$ ), that is, where
TABLE I. Parameters of the tensor terms in units of $\mathrm{MeV} \cdot \mathrm{fm}^{5}$. The $T$ and $U$ values are taken from Refs. [2,4-6], while the values $\alpha$ and $\beta$ are obtained by means of Eq. (5).

there is no approximation in the residual interaction since all its terms are taken into account including the two-body spin-orbit and the two-body Coulomb interactions. In the present case, we also include the tensor terms consistently in $\mathrm{HF}$ and in the residual interaction. The matrix elements of the tensor residual interaction, which are quite involved, have been given in detail in the appendix of Ref. [12]. In our scheme we use box boundary conditions. In other words, the continuum is discretized. In the present case the box radius is set at $15 \mathrm{fm}$ for ${ }^{40} \mathrm{Ca}$ and at $20 \mathrm{fm}$ for ${ }^{208} \mathrm{~Pb}$. The radial step is $0.1 \mathrm{fm}$. 
After solving the HF equations in coordinate space and determining, together with the mean field, the unoccupied states as eigenstates of this mean field in the mentioned large box, we build up a model space of particle-hole (p-h) configurations with given multipolarity $J^{\pi}$, and we solve the RPA matrix equation in that space; basic formulas for RPA calculations are presented in the appendix. The model space includes the configurations built up with all hole states, and with the particle states labeled by $n_{p}, l_{p}$, and $j_{p}$ (number of radial nodes and spatial and total angular momentum): $l_{p}$ and $j_{p}$ take all possible values that are allowed by selection rules, while $n_{p}$ varies between $n_{\max }+1$ ( $n_{\max }$ is the number of nodes of the last occupied state) and $n_{\max }+\Delta n$. The convergence of the RPA solutions is checked in two ways. First, the convergence of the values for the excitation energy and the electromagnetic transition strength $\mathrm{B}(\mathrm{E} \lambda)$ of the $2^{+}$ and $3^{-}$states, as a function of $\Delta n$, is assessed. This is shown in Fig. 1.

As one can see in Fig. 1, the convergence is quite reasonable after $\Delta n=8$, both for the excitation energies and for the transition strengths. We have also checked that the value of $\Delta n=8$ is large enough so that the isoscalar (IS) energy-weighted sum rule for the multipoles $2^{+}, 3^{-}$exhausts practically all (namely, $\approx 97-99 \%$ ) the double commutator value. We take hereafter the value of $\Delta n=8$ for all calculations. In the case of the T46 parameter set, the number of $\mathrm{p}$-h configurations for the multipoles $2^{+}$and $3^{-}$are 1184 and 1416, respectively; the corresponding maximum excitation energies are 109.9 and 116.0 MeV, respectively.

The eigenvalues $E_{n}$ and eigenvectors $|n\rangle$ of the RPA equations allow calculating the response function to different operators. We shall use electric quadrupole and octupole operators,

$$
\hat{F}_{L}=\sum_{i=1}^{Z} e r_{i}^{L} Y_{L M}\left(\hat{r}_{i}\right)
$$
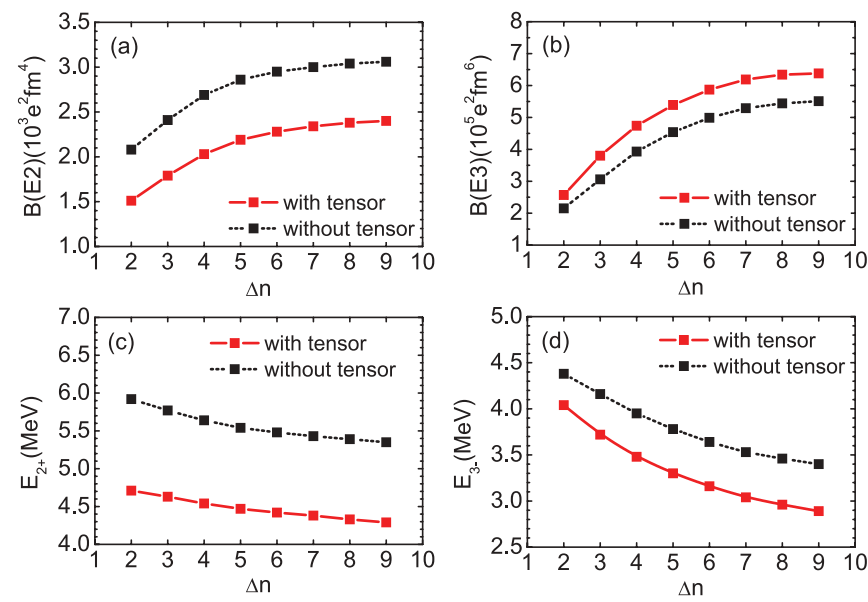

FIG. 1. (Color online) The convergence of the values of $B(E \lambda)$ and of the excitation energies of the lowest collective states in ${ }^{208} \mathrm{~Pb}$. The HF and RPA calculations are performed with and without tensor interactions, using the T46 parameter set. The horizontal axis shows the value of $\Delta n$ (defined in the main text), so that the size of the model space increases from left to right. with $L=2$ and 3, respectively. We will also focus our study on the $1^{+}$states as well. In this case the magnetic dipole transition operator is used, namely,

$$
\hat{F}_{M 1}=\sum_{i=1}^{A}\left\{g_{i}^{s} \vec{\sigma}_{i}+g_{i}^{l} \vec{l}\right\} \frac{e \hbar}{2 m c},
$$

where $g_{p}^{s}=5.586$ and $g_{n}^{s}=-3.826$, respectively, and $g_{p}^{l}=$ 1.0 and $g_{n}^{l}=0.0$ (in units of the nuclear magneton $\mu_{N}=$ $e \hbar / 2 m c)$.

\section{RESULTS}

\section{A. Quadrupole response}

Figure 2 displays the results for the low-lying $2^{+}$state in ${ }^{208} \mathrm{~Pb}$. Even in the case of the TI $J$ forces, the tensor terms are switched on and off artificially to see how much they affect these collective vibrations. In the ground state, the tensor terms change the values of the spin-orbit splittings depending on the
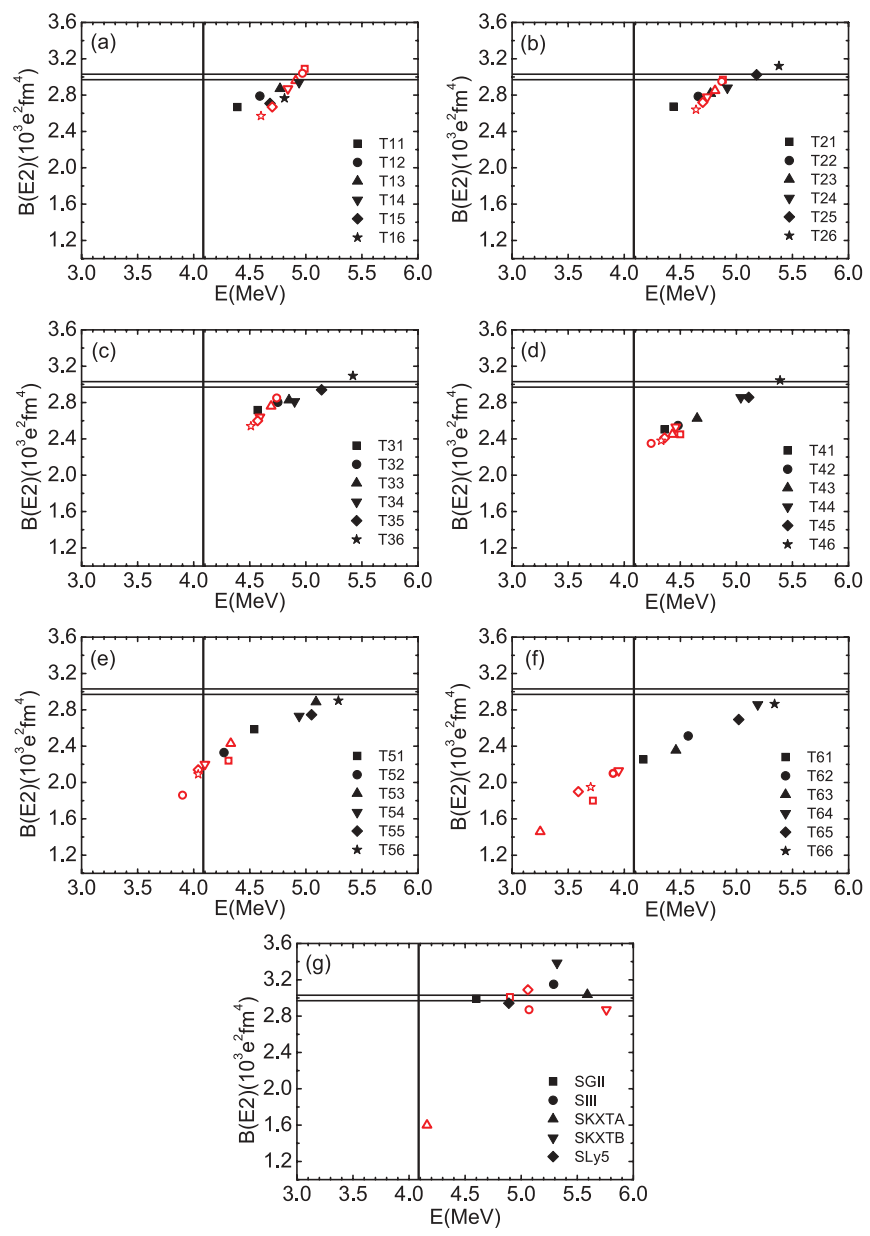

FIG. 2. (Color online) The HF + RPA results for the excitation energy and the $\mathrm{B}(\mathrm{E} 2)$ strength of the lowest quadrupole state in ${ }^{208} \mathrm{~Pb}$. The calculations are performed with and without tensor terms, and the results are denoted by the open and the filled symbols, respectively. The vertical and horizontal lines mark the experimental values with their errors. These experimental data are taken from Ref. [17]. 
spin-orbit densities (3). For the low-lying $2^{+}$state in ${ }^{208} \mathrm{~Pb}$, the main $\mathrm{p}$-h configurations are $1 h_{11 / 2} \rightarrow 2 f_{7 / 2}$ for protons and $1 i_{13 / 2} \rightarrow 2 g_{9 / 2}$ for neutrons. The unperturbed excitation energies depend strongly on the spin-orbit splittings of these configurations. As we can see from Eq. (4), the negative values for $\alpha$ and $\beta$ increase the spin-orbit splitting (since in this case the sign of the second term on the right-hand side is the same as that of the first term in which the derivatives of the densities are negative), while the positive values decrease the splittings. For the TI $J$ families, the effect of tensor terms on the spin-orbit splittings is quite obvious: If the values of $I$ and $J$ are larger than 2, the spin-orbit splittings are decreased, while they tend to increase for $I=1$ (or $J=1$ ) with smaller $J(I)$ values. There are no effects of the tensor terms for $I=2$ and $J=2$ at the mean-field level. In the upper panels of Fig. 2, for the case of T1 $J$ and T $2 J$ interactions having $\beta$ either negative or zero, the spin-orbit splittings are increased so that the excitation energy of the $2^{+}$state is also increased. This effect is very clearly seen in the case of T11. For larger $J$ values and $I=1$, the positive value of $\alpha$ cancels the effect of negative $\beta$, and eventually in the case of $J=6$, the large contribution from the term with $\alpha$ decreases the excitation energy of the $2^{+}$state. As a general trend, in the cases with $I>2$ the excitation energy is pushed down by the tensor effect; the energy is lower for larger $J$ values. This effect is maximum in the case of the T6J family. In the bottom panel, where the results using the interactions in which the tensor terms are added perturbatively are displayed, the tensor effect can be understood essentially by the same argument as that for the TI $J$ family. For example, the tensor effect within SKXTA is similar to the case of T44, and the effect for SKXTB looks like that for T31.

Compared with experimental data marked by the solid lines in Fig. 2, none of the parameter sets give predictions for both the excitation energy and $\mathrm{B}(\mathrm{E} 2)$ value with $10 \%$ accuracies. The T5J parameter sets give quite reasonable results for the empirical excitation energy, while T12, T13, and T21 give values close to the empirical one for the $\mathrm{B}(\mathrm{E} 2)$. If we consider accuracies of the order of $20 \%$ to be still reasonable in comparison with experimental data, several parameter sets satisfy this requirement for both the excitation energy and B(E2) value, namely, T14, T15, T16, T2J, T3J, T41, T43, T44, T45, T53, and SGII. Some parameter sets in the bottom panel of Fig. 2 show reasonable results for the $\mathrm{B}(\mathrm{E} 2)$, but the tensor correlations do not improve the agreement. The $\mathrm{p}$-h matrix elements are shown for two typical configurations contributing to the low-lying $2^{+}$state, namely, $\left(2 \mathrm{f}_{7 / 2} 1 \mathrm{~h}_{11 / 2}{ }^{-1}\right)_{\pi}$ and $\left(2 \mathrm{~g}_{9 / 2} 1 \mathrm{i}_{13 / 2}{ }^{-1}\right)_{\nu}$. The tensor matrix elements are relatively small in comparison with the central part of the Skyrme interactions, since they are at most $200 \mathrm{keV}$. Thus, the effect of the tensor interaction in the RPA matrix is small. For reference, the values of the two-body spin-orbit and Coulomb matrix elements are also shown in Table II. These are even smaller than the tensor matrix elements.

In summary for this section, the behavior of the energy of the low-lying $2^{+}$state can be fairly well understood. As was

TABLE II. The p-h RPA matrix elements $A(B)$ of Eq. (A6) for two typical configurations coupled to $2^{+}$in ${ }^{208} \mathrm{~Pb}$. The p-h energy difference $\varepsilon_{p}-\varepsilon_{h}$ is not included in the $A$ matrix here. The symbols $\mathbf{M}_{\text {central }}, \mathbf{M}_{\mathrm{so}}, \mathbf{M}_{\text {Coul }}$, and $\mathbf{M}_{\text {tensor }}$ denote the central part (proportional to $t_{0}, t_{1}, t_{2}$, and $t_{3}$ ), the two-body spin-orbit, the Coulomb part, and the tensor parts (triplet-even and triplet-odd), respectively.

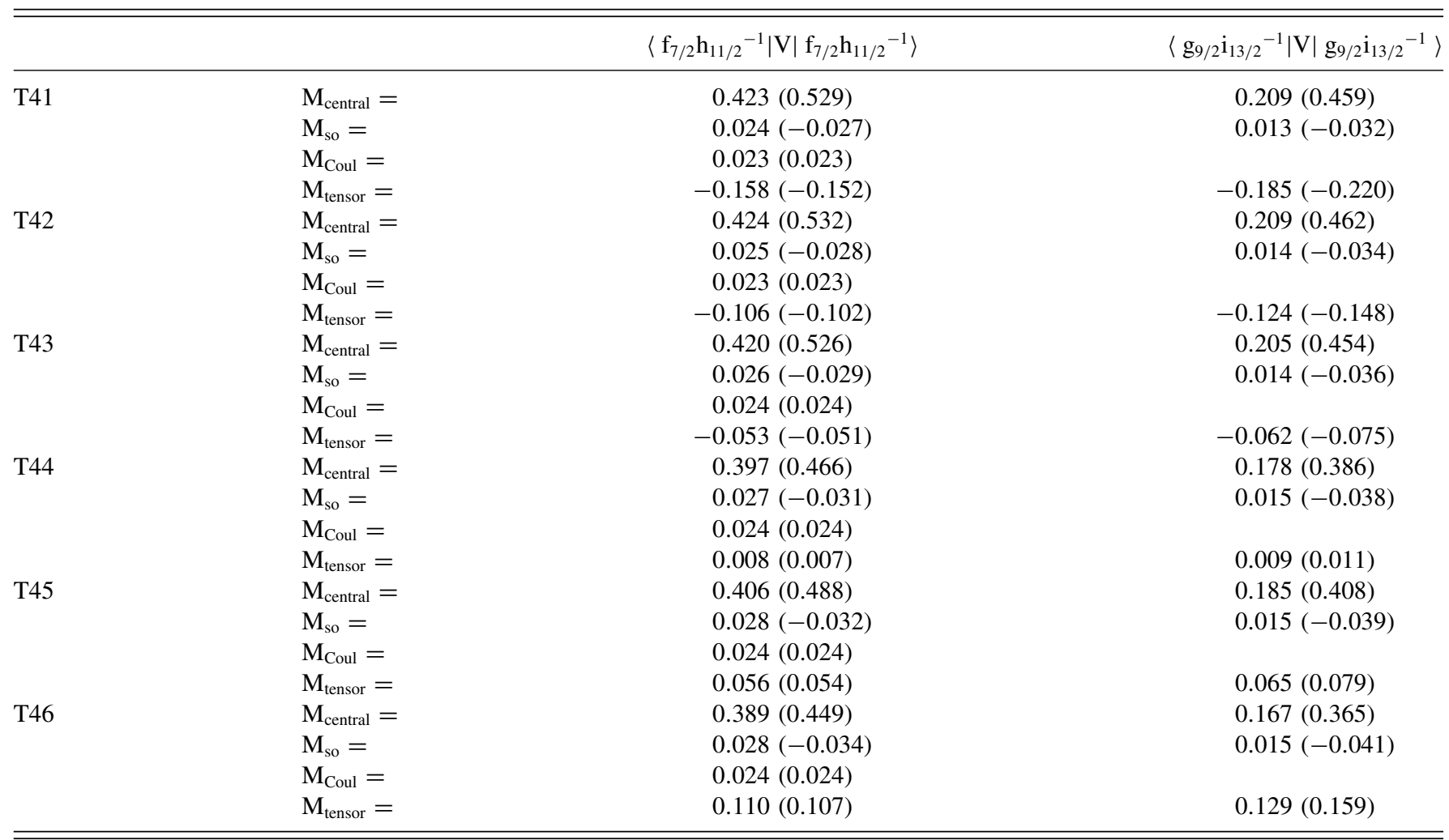


already noticed in Ref. [12], tensor effects are mainly related to the mean field since the residual tensor interaction matrix elements are either tiny or not very large, as shown here in Table II.

\section{B. Octupole response}

In a similar style as for the quadrupole case, the results for the lowest octupole state of ${ }^{208} \mathrm{~Pb}$ are displayed in Fig. 3 . At variance with the quadrupole case, several configurations in addition to the two major configurations, $\left(1 \mathrm{~h}_{9 / 2} 2 \mathrm{~d}_{3 / 2}{ }^{-1}\right)_{\pi}$ and $\left(\lg _{9 / 2} 3 \mathrm{p}_{3 / 2}{ }^{-1}\right)_{\nu}$, contribute to the collective low-lying $3_{1}^{-}$ state. This collectivity is enough, so that the effects coming from unperturbed configurations that are pushed upward or downward by the tensor terms, together with the effect of the tensor residual interaction, tend to cancel one another. The $\mathrm{p}-\mathrm{h}$ tensor matrix elements $\mathrm{M}_{\text {tensor }}$ displayed in Table III (which is analogous to Table II, which has been discussed above) are much smaller than those of central part of the Skyrme
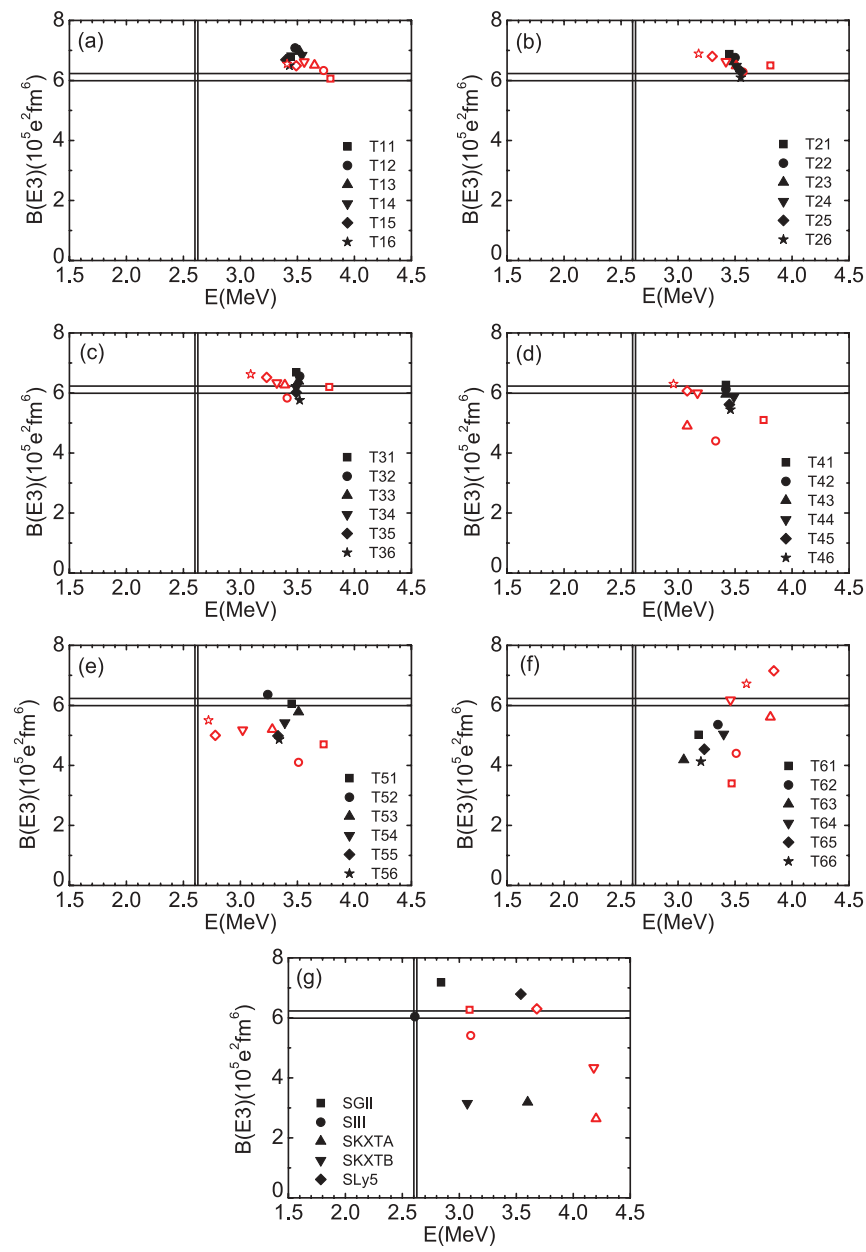

FIG. 3. (Color online) The HF + RPA results for the excitation energy and the $\mathrm{B}(\mathrm{E} 3)$ strength of the lowest octupole state in ${ }^{208} \mathrm{~Pb}$. The calculations are performed with and without tensor terms, and the results are denoted by the open and the filled symbols, respectively. The vertical and horizontal lines mark the experimental values with their errors. These experimental data are taken from Ref. [18]. interaction $\mathrm{M}_{\text {central }}$ and are typically less than $100 \mathrm{keV}$ : It should be noted that the tensor matrix elements in the $3^{-}$ case are even smaller (by a factor $2 \sim 3$ ) than those displayed in Table II for the $2^{+}$case. From Fig. 3 one can see in general that the energies and $\mathrm{B}(\mathrm{E} 3)$ values in ${ }^{208} \mathrm{~Pb}$ are not affected significantly when a tensor is included, in the case of the TI $J$ sets with $I \leqslant 4$ and $J \leqslant 4$. The tensor effect is larger for larger $I, J$ values. However, the effect of tensor correlations is not straightforward compared with the case of the $2^{+}$state, since not only the spin-orbit splittings but also the shell spacing between the even parity and the odd parity states around the Fermi energy change the excitation energy and the collectivity. In comparison with experiment, the T55 and T56 sets give quite reasonable results in terms of both the excitation energy and the $\mathrm{B}(\mathrm{E} 3)$ value. Among the interactions with tensor terms in the bottom panel, SGII and SIII give predictions close to the experimental data. Putting together the quadrupole and octupole results, we note that the T36, T44, T45, T46, and SGII parameter sets provide accuracies on the order of $20 \%$ in both cases.

The low-lying $3^{-}$state also exists in ${ }^{40} \mathrm{Ca}$. In order to check how the shell spacing around the Fermi surface is relevant in this case, we study the octupole vibration in ${ }^{40} \mathrm{Ca}$ and report our results in Fig. 4. For this specific aim, we focus on the parameter sets T4J (because some of them have been shown to perform well for quadrupole and octupole in ${ }^{208} \mathrm{~Pb}$ ) and on the interactions with perturbative tensor terms. In Table IV, the HF energy differences between the occupied positive parity states $1 \mathrm{~d}_{3 / 2}$ and $2 \mathrm{~s}_{1 / 2}$, and the unoccupied negative parity state $1 f_{7 / 2}$ state, are shown with and without the contribution from the tensor terms. Notice that the tensor interactions have essentially a negligible effect on the single-particle energies in the spin-orbit saturated nucleus ${ }^{40} \mathrm{Ca}$. One can see that, because of the significantly low (high) energies of the $j_{>}\left(j_{<}\right)$ orbitals, some parameter sets are characterized by an energy gap around the Fermi surface that is too small. This tendency is clear for SKXTB and for T4 $J$ sets with large $J$, in particular T46. Because of these small energy gaps, the lowest $3^{-}$state is almost degenerate with the ground state in the case of T46, and RPA is unstable (the lowest state has an associated solution with nonzero imaginary part) in the case of SKXTB. We have noticed that this anomaly for the excitation energy of the lowest $3^{-}$state shows up for other members such as the T1 $J$ and T2 $J$ families, although the behavior of the tensor force differs in detail. On the other hand, some members of TI $J$ family give reasonable results for $\mathrm{B}(\mathrm{E} 3)$ and the excitation energies of $3^{-}$in ${ }^{40} \mathrm{Ca}$ as can be seen in the upper panels in Fig. 4. In particular, T33, T34, T35,T43, T44, and T45, together with SGII and SLy5, give results that agree within a $20 \%$ accuracy with the experimental data.

The shell gaps in ${ }^{40} \mathrm{Ca}$ produced by some of the TIJ interactions (such as SKTB or T4J with large $J$ ) turn out to be much smaller $(1 \sim 3 \mathrm{MeV})$ than those associated with standard Skyrme forces such as SGII and SIII, which are fitted without tensor terms. This explains the small excitation energy of the low-lying $3^{-}$in ${ }^{40} \mathrm{Ca}$. This decrease of the energy gaps associated with some sets of the TIJ family with respect to other forces does not appear in the case of ${ }^{208} \mathrm{~Pb}$ because of the effect of the RPA tensor correlations. Thus, this shell-spacing 
TABLE III. The same as Table II for two typical configurations coupled to $3^{-}$in ${ }^{208} \mathrm{~Pb}$.

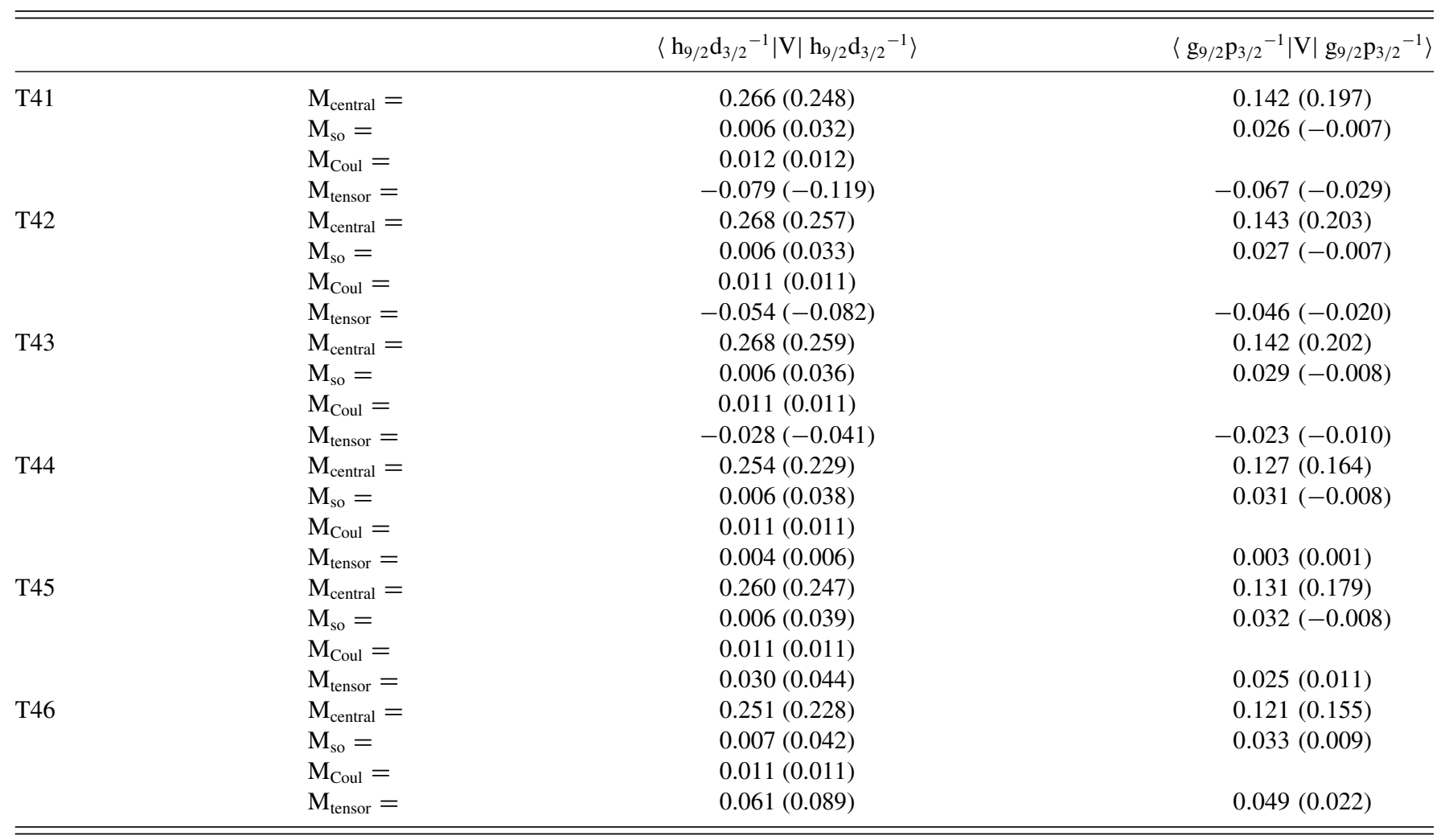

problem in the $\mathrm{Ca}$ region remains an open issue for future fittings of the Skyrme parameters including tensor terms.

\section{Magnetic dipole response}

On quite general grounds, it can be expected that the effects of the tensor force are larger for spin (or for spinisospin) states, both at the mean-field level, since unperturbed configurations are sensitive to the spin-orbit splittings, and
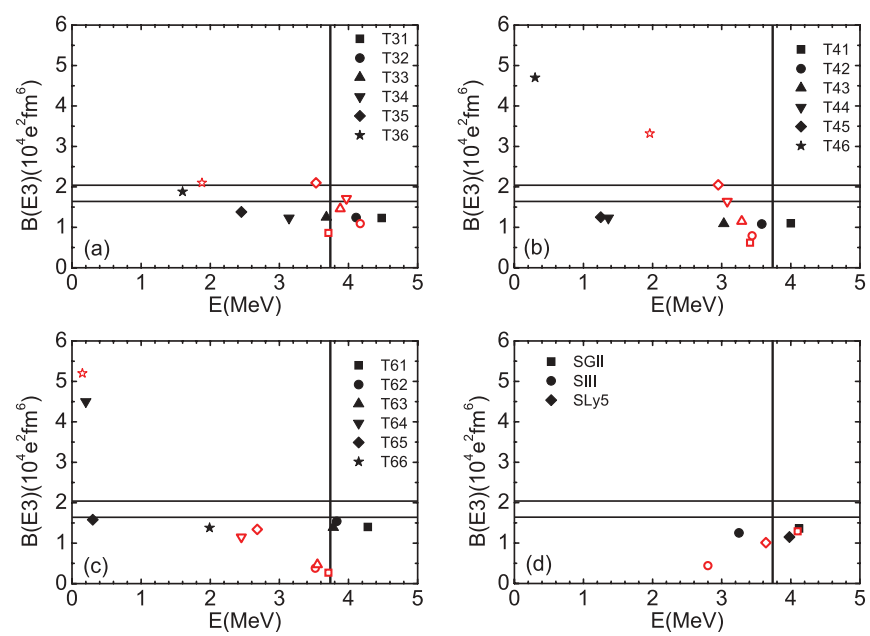

FIG. 4. (Color online) The same as Fig. 3 for the 3 -state in ${ }^{40} \mathrm{Ca}$. Shown are selected results of T3J, T4J, T6J, SGII, SIII, and SLy5 interactions. Experimental data are taken from Ref. [18]. also as far as RPA correlations are concerned [19]. We analyze in this subsection the results for the $1^{+}$magnetic dipole excitations in ${ }^{208} \mathrm{~Pb}$, which are reported in Fig. 5. In the unperturbed spectrum, the main role is played by the proton $1 \mathrm{~h}_{11 / 2} \rightarrow 1 \mathrm{~h}_{9 / 2}$ and neutron $1 \mathrm{i}_{13 / 2} \rightarrow 1 \mathrm{i}_{11 / 2}$ configurations. In general, the proton configuration has lower energy (by about $2 \mathrm{MeV}$ ) than the neutron configuration due to a smaller angular momentum. As discussed for the low quadrupole state, when tensor terms are introduced, the spin-orbit splittings are increased in the case of sets of the TIJ family when $J \leqslant 3$ (negative $\alpha$ or small $\beta$ values), while the spin-orbit splittings are decreased in the case of sets with $J \geqslant 3$ (positive $\alpha$ and $\beta$ values). This feature is enhanced for larger $I$ cases (positive $\beta$ ). All this can be easily understood from Eq. (4).

The values of the excitation energies in Fig. 5 reflect this behavior of the spin-orbit splittings. Concerning the transition strengths, we notice that for large $J$ (i.e., $J \geqslant 5$ ) combined with $I \geqslant 2$, the high-energy $1^{+}$state has larger B(M1) strength than that of the low-energy state. This fact is known empirically as the isovector dominance in the magnetic transitions [21,22], and those interactions show properly this specific feature of those transitions. On the other hand, the parameter sets with $J \leqslant 3$ fail to provide the isovector dominance feature. Among the parameter sets in the last panels of Fig. 5, SKXTA and SKXTB show the isovector dominance feature, while the other three parameter sets fail to reproduce this property. It can be noticed that SGII and SIII give quite reasonable descriptions of $1^{+}$states without the tensor terms, even quantitatively in the case of the excitation energies; however, the good properties 
TABLE IV. The energy difference between the HF states, $\varepsilon_{1 f_{7 / 2}}-\varepsilon_{2 s_{1 / 2}}$ and $\varepsilon_{1 f_{7 / 2}}-\varepsilon_{1 d_{3 / 2}}$, in the nucleus of ${ }^{40} \mathrm{Ca}$ (in MeV).

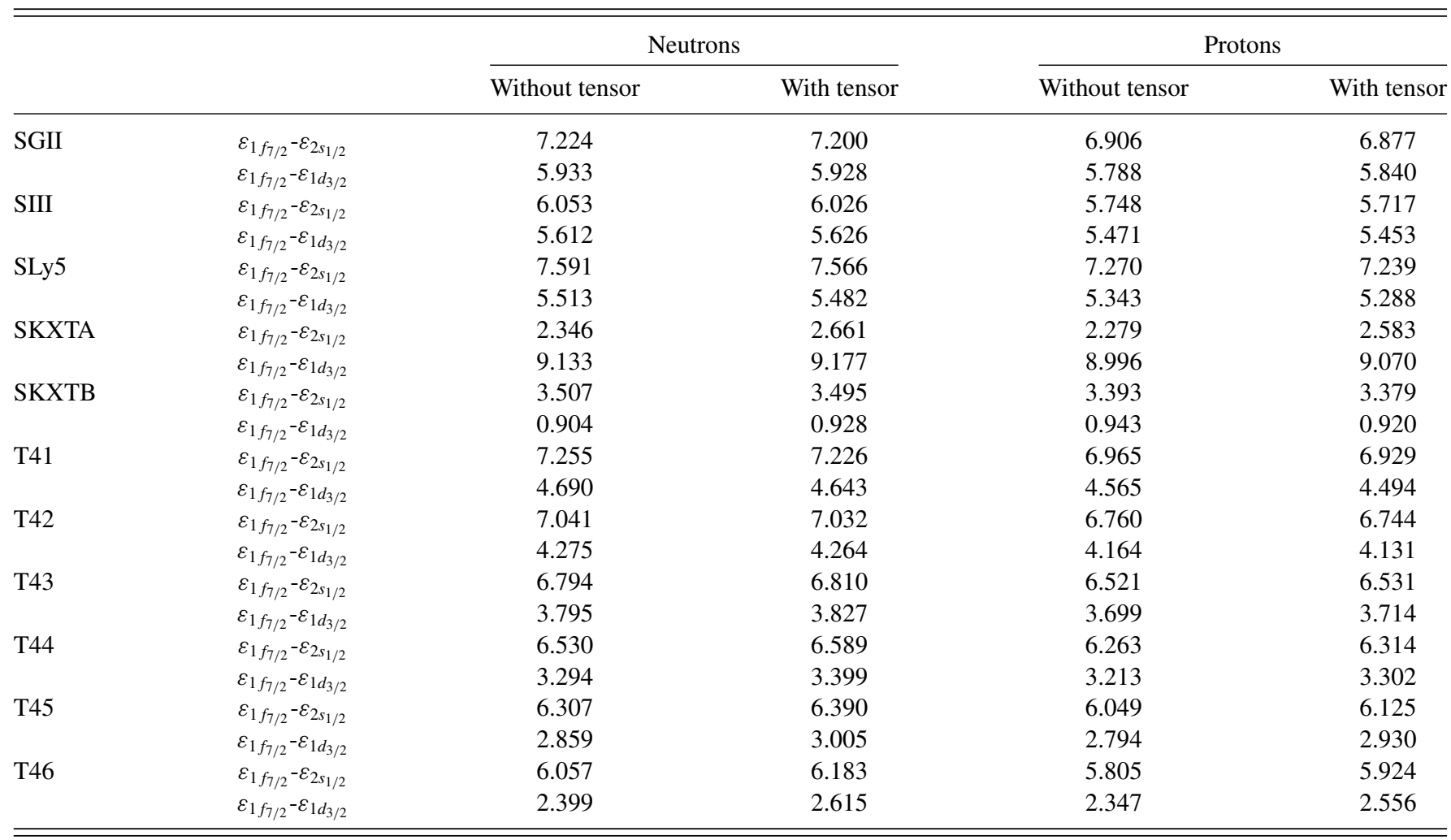

of the $\mathrm{B}(\mathrm{M} 1)$ values are destroyed by the inclusion of tensor terms in the case of both SGII and SIII.

Since the values of $\alpha$ and $\beta$ have different sign and similar magnitude in both the SGII and SIII cases, the tensor effects are largely canceled at the mean-field level so that the RPA energies have also not been shifted significantly. However, the B(M1) values are drastically changed by the tensor correlations, and the low-energy $1^{+}$state has much larger strength than that of the high-energy $1^{+}$state. The same features can be seen in the B(M1) values obtained with the parameter sets T51, T52, and T61, which have values of $T$ and $U$ close to those of SGII (in both sign and magnitude). The p-h two-body matrix elements $A$ and $B$ in Eq. (A6) for the $\mathrm{T} 4 \mathrm{~J}$ interactions are given in Table $\mathrm{V}$ for the central terms, two-body spin-orbit, and tensor terms, respectively. As was shown in Ref. [20], the antisymmetrized tensor p-h matrix elements are proportional to $T$ and $U$ parameters and read

$$
V_{T, A S}^{\lambda}=\left(\frac{1}{2} a_{\lambda} T+\frac{3}{2} b_{\lambda} U\right)+\left(-\frac{1}{2} a_{\lambda} T+\frac{1}{2} b_{\lambda} U\right)\left\langle\tau_{z 1} \cdot \tau_{z 2}\right\rangle .
$$

The matrix elements (10) are different according to the values of $(p h, q)$ and $\left(p^{\prime} h^{\prime}, q^{\prime}\right)$ : If $q=q^{\prime}$, namely, for protonproton or neutron-neutron $\mathrm{p}-\mathrm{h}$ interaction, the matrix elements become

$$
V_{T, A S}^{\lambda}=2 b_{\lambda} U
$$

while Eq. (10) becomes

$$
V_{T, A S}^{\lambda}=a_{\lambda} T+b_{\lambda} U
$$

in the case $q \neq q^{\prime}$, namely, between proton ( $\left.p h\right)$ and neutron $\left(p^{\prime} h^{\prime}\right)$ excitations. The first two matrix elements reported in Table V correspond to Eq. (11), whereas the last column of the same table can be interpreted by means of Eq. (12). The signs of $a_{\lambda}$ and $b_{\lambda}$ should be negative for $1^{+}$excitations [as in Eq. (5) of Ref. [20], with appropriate quantum numbers $\lambda=\lambda^{\prime}=1$ ].

As is expected from the values of $T$ and $U$ in Table I, the diagonal $A$ and $B$ matrix elements for the $\mathrm{h}_{9 / 2} \mathrm{~h}_{11 / 2}{ }^{-1}$ and $\mathrm{i}_{11 / 2} \mathrm{i}_{13 / 2}{ }^{-1}$ configurations even change drastically their sign, while the off-diagonal one between these two configurations changes only modestly, since the sum of $T$ and $U$ is rather slowly varying, as can be seen in Table I. Negative matrix elements enhance the isovector (IV) dominance of the higher excited $1^{+}$state and at the same time the IS dominance of the lower excited $1^{+}$state (cf. the example of T45 and T46 in Table VI). Alternatively, positive matrix elements decrease the isovector character of the higher excited $1^{+}$state and decrease its B(M1) strength (cf. the example of T41 and T42 in Table VI). Since the $T$ and $U$ values associated with SGII, SIII, and SLy5 in Table I are almost of the same magnitude as those of T61 and T62, the same features appear in the results for the B(M1) values in the bottom panels of Fig. 5 .

In the case of SLy5 plus the tensor parameters of Ref. [4], the spin-orbit splittings are increased when the tensor terms are taken into account. In RPA, without tensor, we have found two peaks at 7.39 and $9.14 \mathrm{MeV}$. The low- (high-) energy state has more IV (IS) character. Since experimentally one finds the isovector dominance mentioned above, namely, the low(high-) energy $1^{+}$state has more IS (IV) character, there is an inversion that is related to the values of the Landau parameters 
TABLE V. The same as Tables II and III for three typical configurations coupled to $1^{+}$in ${ }^{208} \mathrm{~Pb}$. The $\mathrm{h}_{9 / 2} \mathrm{~h}_{11 / 2}{ }^{-1}\left(\mathrm{i}_{11 / 2} \mathrm{i}_{13 / 2}{ }^{-1}\right)$ is a proton (neutron) configuration.

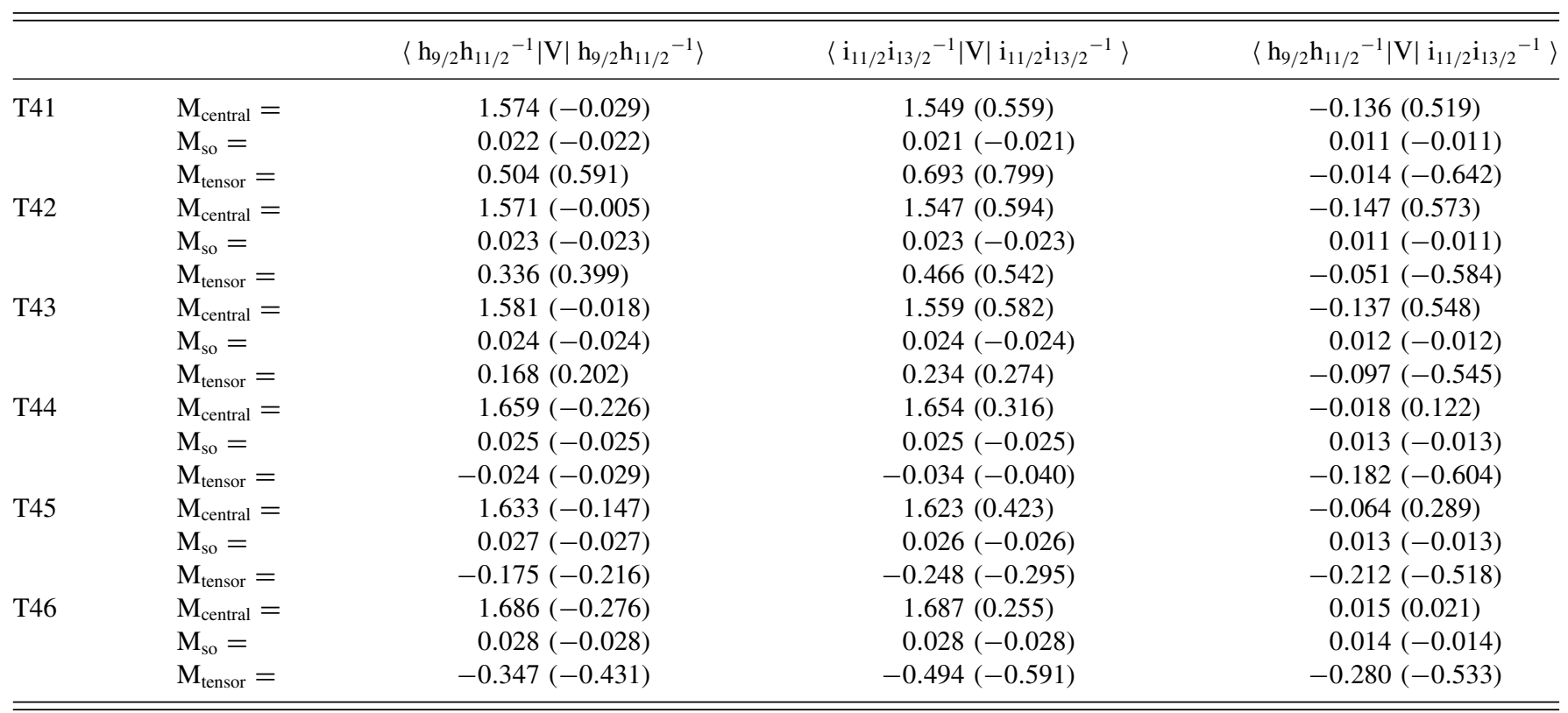

$G_{0}$ and $G_{0}^{\prime}$, or ultimately to the unconstrained and not fully satisfactory spin properties of the SLy5 interaction. We notice that inversions of IS and IV spin doublet have also emerged from the self-consistent Gogny calculations of Ref. [23]. It was noticed in our previous work [12] that the matrix elements of the residual tensor force are rather small $(\approx-250 \mathrm{keV})$ in the case of SLy5 plus perturbative tensor terms. The results in ${ }^{208} \mathrm{~Pb}$ obtained here by using the $\mathrm{T} I J$ sets with $I \geqslant 4$ and $J \geqslant 4$ seem better. In particular, we can notice that in this case one has the correct ordering in the spin doublet, the IS (IV)

TABLE VI. The RPA amplitudes $X$ and $Y$ for the main $1^{+}$states in ${ }^{208} \mathrm{~Pb}$.

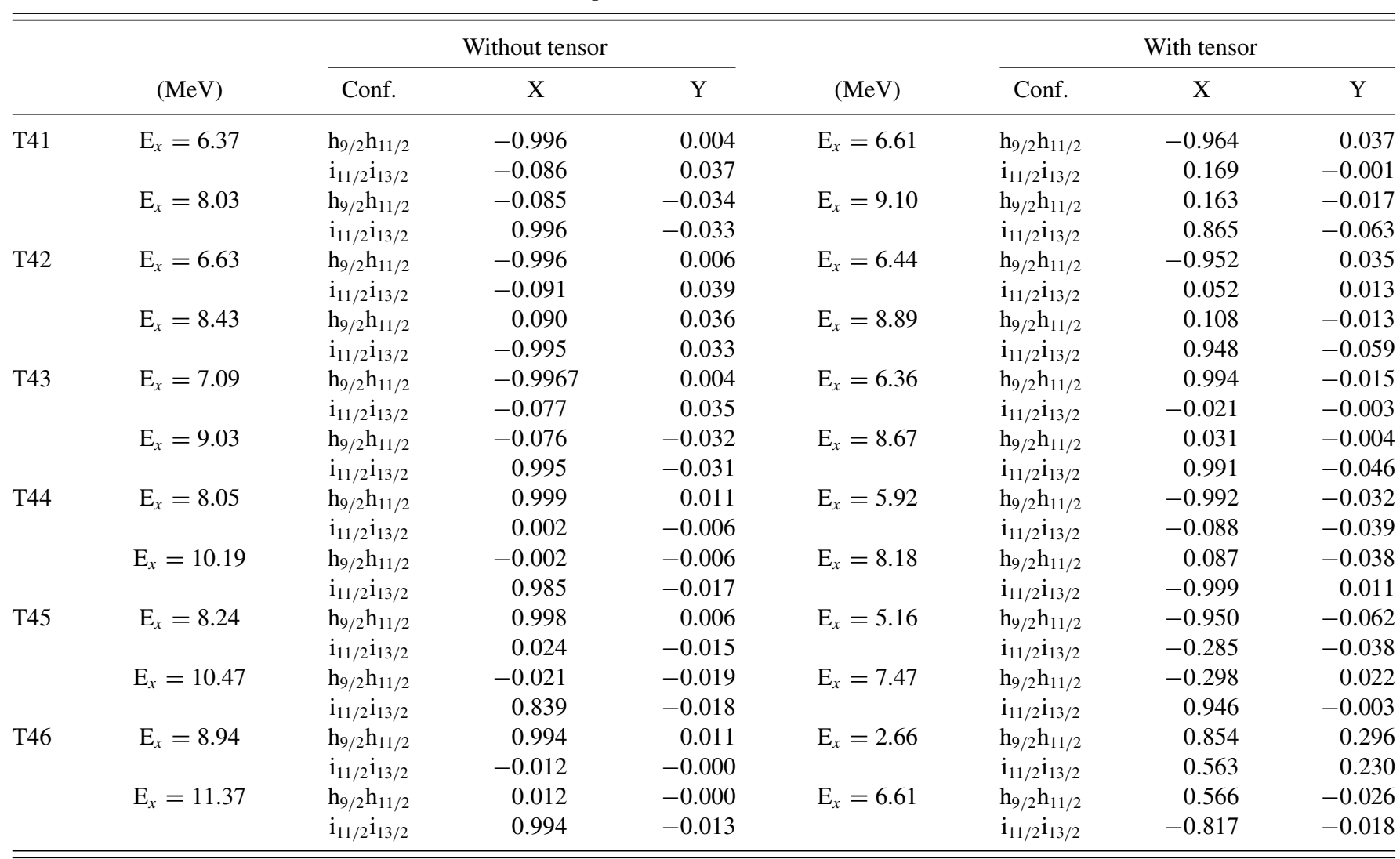



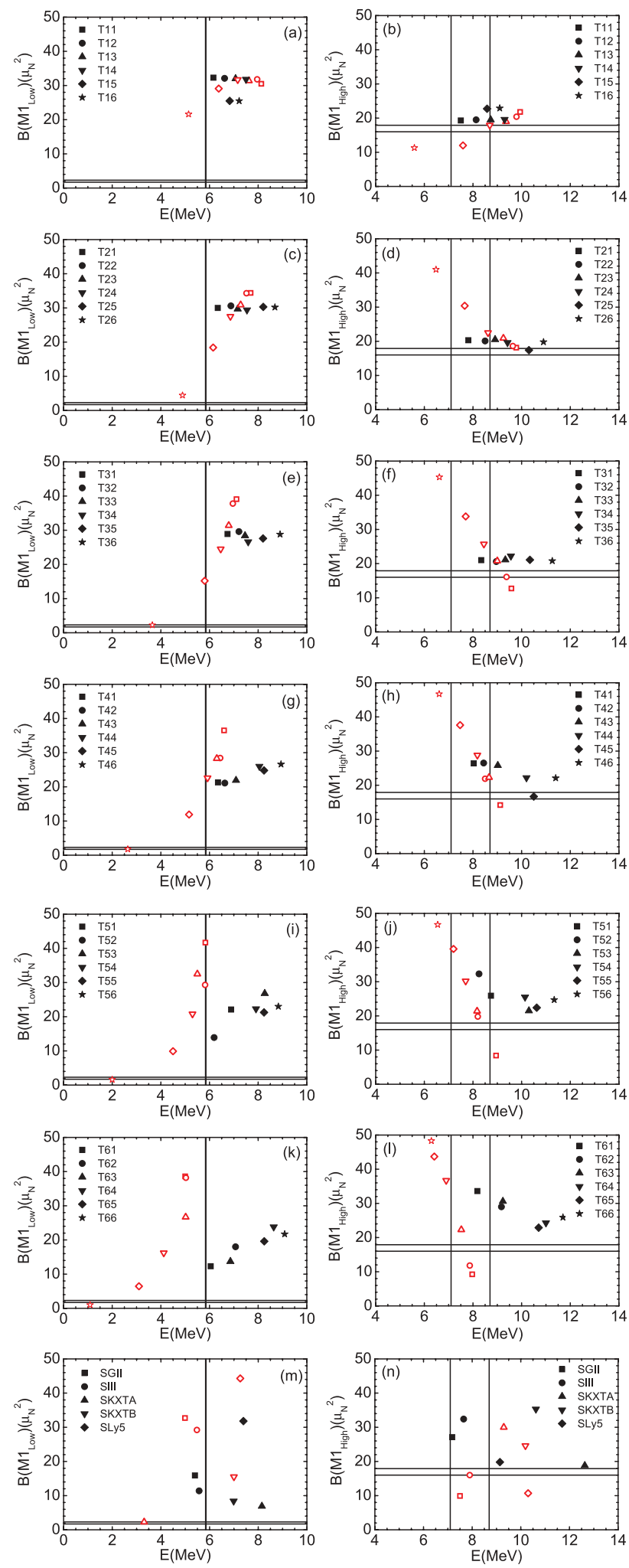

FIG. 5. (Color online) The HF+ RPA results for the excitation energy and the B(M1) strength of the low (left panels) and high (right panels) $1^{+}$state in ${ }^{208} \mathrm{~Pb}$. The calculations are performed with and without tensor terms, and the results are denoted by the open and the filled symbols, respectively. The vertical and horizontal lines mark the experimental values with their errors. These experimental data are taken from Ref. [22]. state being the low- (high) energy state. In fact, with the values of the parameter sets $T$ and $U$ associated with the interactions with $I \geqslant 4$ and $J \geqslant 4$, namely, with positive and large $T$ and positive or almost zero $U$ values, the IS and IV characters of the states are more pronounced due to a larger positive value of the matrix element mixing neutrons and protons. Thus the drastic changes of the B(M1) strength in Fig. 5 are caused by the nature of the $\mathrm{p}-\mathrm{h}$ matrix elements in the RPA equation.

\section{SUMMARY}

In this paper we deal with the effects produced by the tensor terms on the multipole response of finite nuclei ${ }^{208} \mathrm{~Pb}$ and ${ }^{40} \mathrm{Ca}$. We have attempted to disentangle the effects due to the modifications of the static mean field, and those due to the residual interaction, by analyzing the results of HF plus RPA calculations performed in the case of quadrupole, octupole, and magnetic dipole channels. In particular, we have analyzed in a systematic and careful way the performance of the interactions of the TIJ family. Our HF-RPA scheme includes all terms self-consistently, in particular the tensor-even and tensor-odd terms, and the calculations have been performed in large model spaces.

The modifications of the static mean field had been already understood by means of our previous work. The dominant character (attractive or repulsive) of the residual interaction matrix elements has been extracted from the numerical calculations and understood on the basis of a separable approximation for the tensor $\mathrm{p}$-h force. Then, it has become evident that since these two effects are governed by different combinations of the parameters of the tensor force, the effects that are visible in the final results are ruled by a delicate interplay and are nontrivial. This is one of the main findings of the present work.

We found out that $2^{+}$and $3^{-}$states are better described by some Skyrme parameter sets with tensor terms such as T36, T44, T45, T46, and SGII interactions in terms of both the excitation energies and the transition strength. The parameter sets T44, T45, and SGII also give reasonable results for $3^{-}$in ${ }^{40} \mathrm{Ca}$. This provides us with a promising background for further applications of the particle-vibration coupling model.

The excitation energies of magnetic dipole states are much affected by the inclusion of the tensor terms at the mean-field level, since unperturbed configurations correspond exactly to transition energies between spin-orbit partners. The inclusion of the tensor residual interaction is demanded by self-consistency: For its matrix elements we have extracted typically values around a few or several hundreds of $\mathrm{keV}$, but these values depend, of course, on the values of the tensor force parameters $T$ and $U$. It is noticed that the p-h residual interactions can change drastically the isospin properties of the two $1^{+}$states. Namely, a larger positive $U$ value enhances the IV (IS) character of the high- (low-) energy $1^{+}$state. On the other hand, a combination of positive large value of $T$ and a negative value of $U$ decreases the IV character of the high excited state and increases that of the low excited state. 
To fit an ultimate Skyrme set including the tensor terms, one should ask the question whether the Skyrme ansatz is general enough for the spin-isospin channels. Some of the most modern, and most widely used, sets such as the Lyon parametrizations have somewhat unsatisfactory values of the spin and spin-isospin Landau parameters, and this anomaly should be cured. Improved Skyrme sets fitted with the tensor terms can provide some possibility of curing this drawback, as far as their performance for excited states is concerned. We feel the need that Skyrme parameter sets are fitted with protocols that do include not only the ground-state properties but also the excited-state properties (for instance, through constraints on the Landau parameters). This is one of the main perspectives opened by our present work, which goes together with other efforts aimed at fixing the appropriate tensor terms of zerorange forces such as those of Refs. [6,24].

\section{ACKNOWLEDGMENTS}

This work is partially supported by the Japanese Ministry of Education, Culture, Sports, Science, and Technology by a Grant-in-Aid for Scientific Research with the program number [C (2)] 20540277. CL acknowledges the support of the National Science Foundation of China with Grant Nos. 10875150. GC acknowledges partial support from the Italian research project (PRIN) named "Many-body theory of nuclear systems and implications on the physics of neutron stars".

\section{APPENDIX: BASIC FORMULAS FOR RPA CALCULATIONS}

We summarize the basic formulas of the RPA calculations in this appendix. The particle-hole (p-h) state with angular momentum $\lambda, \mu$ is defined by

$$
A_{p h, \alpha}^{\dagger}(\lambda, \mu)=\sum_{m_{p}, m_{h}}\left(j_{h} j_{p} m_{h} m_{p} \mid \lambda, \mu\right) a_{j_{p}, m_{p}}^{\dagger} a_{\overline{j_{h}, m_{h}}},
$$

where $\alpha=\nu, \pi$ denotes neutrons and protons and $a_{\overline{j_{h}, m_{h}}}=$ $(-)^{j_{h}-m_{h}} a_{j_{h},-m_{h}}$, respectively. The RPA phonon operator of $n$th excited state is defined by

$$
Q_{n}^{\dagger}(\lambda, \mu)=\sum_{p, h, \alpha=\mu, \pi} X_{p h, \alpha}^{n} A_{p h, \alpha}^{\dagger}(\lambda, \mu)+Y_{p h, \alpha}^{n} A_{p h, \alpha}(\lambda,-\mu) .
$$

This definition of phonon operator leads to the transition amplitudes

$$
\begin{aligned}
\left\langle n: \lambda, \mu\left|\hat{F}_{\lambda, v}\right| 0\right\rangle= & \frac{1}{\sqrt{2 \lambda+1}} \sum_{p, h, \alpha}\left\langle p\left\|\hat{F}_{\lambda}\right\| h\right\rangle_{\alpha} \\
& \times\left(X_{p h, \alpha}^{n}-c Y_{p h, \alpha}^{n}\right),
\end{aligned}
$$

where $\left\langle p\left\|\hat{F}_{\lambda}\right\| h\right\rangle$ is the reduced matrix element and $c$ is the phase factor for $\mathrm{p}-\mathrm{h}$ conjugation [25],

$$
c=\left\{\begin{aligned}
1 & \text { for } \quad \mathbf{s} f(\mathbf{r}), \quad \mathbf{l} f(\mathbf{r}), \quad \mathbf{p} \\
-1 & \text { for } \quad f(\mathbf{r}), \quad(\mathbf{l} \cdot \mathbf{s}) f(\mathbf{r}),
\end{aligned}\right.
$$

where $f(\mathbf{r})$ is any function of the position $\mathbf{r}$. The RPA matrix equations are given by

$$
\left(\begin{array}{cc}
A & B \\
B^{*} & A^{*}
\end{array}\right)\left(\begin{array}{l}
X^{n} \\
Y^{n}
\end{array}\right)=\hbar \omega_{n}\left(\begin{array}{cc}
1 & 0 \\
0 & -1
\end{array}\right)\left(\begin{array}{l}
X^{n} \\
Y^{n}
\end{array}\right),
$$

where $\hbar \omega_{n}$ is the excitation energy of the $n$th excited state. Thematrix elements $A$ and $B$ read

$$
\begin{aligned}
A_{p h, \alpha ; p^{\prime} h^{\prime}, \alpha^{\prime}}= & \left(\varepsilon_{p}-\varepsilon_{h}\right) \delta_{p, p^{\prime}} \delta_{h, h^{\prime}} \delta_{\alpha, \alpha^{\prime}} \\
& +\left\langle(p h) \lambda \mu, \alpha|V|\left(p^{\prime} h^{\prime}\right) \lambda \mu, \alpha^{\prime}\right\rangle, \\
B_{p h, \alpha ; p^{\prime} h^{\prime}, \alpha^{\prime}}= & \left\langle(p h) \lambda \mu, \alpha ;\left(p^{\prime} h^{\prime}\right) \overline{\lambda \mu}, \alpha^{\prime}|V| 0\right\rangle .
\end{aligned}
$$

[1] M. Bender, P.-H. Heenen, and P.-G. Reinhard, Rev. Mod. Phys. 75, 121 (2003).

[2] B. A. Brown, T. Duguet, T. Otsuka, D. Abe, and T. Suzuki, Phys. Rev. C 74, 061303(R) (2006).

[3] J. Dobaczewski, in Proceedings of the Third ANL/MSU/JINA/INT RIA Workshop, edited by T. Duguet, H. Esbensen, K. M. Nollett, and C. D. Roberts (World Scientific, Singapore, 2006), p. 152.

[4] G. Colò, H. Sagawa, S. Fracasso, and P. F. Bortignon, Phys. Lett. B 646, 227 (2007) [see also ibid. 668, 457 (2008)].

[5] D. M. Brink and F. Stancu, Phys. Rev. C 75, 064311 (2007)

[6] T. Lesinski, M. Bender, K. Bennaceur, T. Duguet, and J. Meyer, Phys. Rev. C 76, 014312 (2007).

[7] M. Grasso, Z. Y. Ma, E. Khan, J. Margueron, and N. Van Giai, Phys. Rev. C 76, 044319 (2007).

[8] W. Zou, G. Colò, Z. Ma, H. Sagawa, and P. F. Bortignon, Phys. Rev. C 77, 014314 (2008).

[9] M. Zalewski, J. Dobaczewski, W. Satula, and T. R. Werner, Phys. Rev. C 77, 024316 (2008).
[10] T. Otsuka, T. Suzuki, R. Fujimoto, H. Grawe, and Y. Akaishi, Phys. Rev. Lett. 95, 232502 (2005); T. Otsuka, T. Matsuo, and D. Abe, ibid. 97, 162501 (2006).

[11] J. Li, G. Colò, and J. Meng, Phys. Rev. C 78, 064304 (2008).

[12] Li-Gang Cao, G. Colò, H. Sagawa, P. F. Bortignon, and L. Sciacchitano, Phys. Rev. C 80, 064304 (2009).

[13] G. Colò, H. Sagawa, and P. F. Bortignon, Phys. Rev. C 82, 064307 (2010).

[14] T. H. R. Skyrme, Nucl. Phys. 9, 615 (1959).

[15] G. Colò, P. F. Bortignon, S. Fracasso, and N. Van Giai, Nucl. Phys. A 788, 173c (2007).

[16] L. Trippa, G. Colò, and E. Vigezzi, Phys. Rev. C 77, 061304(R) (2008)

[17] S. Raman et al., Atom. Data Nucl. Data Tables 78, 1 (2001).

[18] T. Kibédi and R. H. Spear, Atom. Data Nucl. Data Tables 80, 35 (2002) 
[19] C. L. Bai, H. Sagawa, H. Q. Zhang, X. Z. Zhang, G. Colò, and F. R. Xu, Phys. Lett. B 675, 1 (2009); C. L. Bai, H. Q. Zhang, X. Z. Zhang, F. R. Xu, H. Sagawa, and G. Colò, Phys. Rev. C 79, 041301(R) (2009).

[20] C. L. Bai, H. Q. Zhang, H. Sagawa, X. Z. Zhang, G. Colò, and F. R. Xu, Phys. Rev. Lett. 105, 072501 (2010).

[21] A. Bohr and B. R. Mottelson, Nuclear Structure, Vol. II (W. A. Benjamin, Reading, MA, 1975), p. 638.
[22] T. Shizuma, T. Hayakawa, H. Ohgaki, H. Toyokawa, T. Komatsubara, N. Kikuzawa, A. Tamii, and H. Nakada, Phys. Rev. C 78, 061303(R) (2008).

[23] V. De Donno, G. Có, C. Maieron, M. Anguiano, A. M. Lallena, and M. Moreno Torres, Phys. Rev. C 79, 044311 (2009).

[24] D. Davesne, M. Martini, K. Bennaceur, and J. Meyer, Phys. Rev. C 80, 024314 (2009).

[25] A. Bohr and B. R. Mottelson, Nuclear Structure, Vol. I (W. A. Benjamin, Reading, MA, 1969). 